\title{
"Allumeeez le fun » : le jeu de mots comme lieu de croisement des dynamiques linguistique et sociolinguistique dans le discours publicitaire
}

Résumé : Le jeu de mots est, au moins dans le domaine publicitaire, un écart volontaire aux normes linguistiques de la part du locuteur, dans le but d'interpeller d'abord et de faire (sou)rire son interlocuteur ensuite. Ces dimensions phatique mais aussi esthético-ludique suffisent à justifier l'utilisation quasi systématique de ce mécanisme linguistique par les annonceurs. Pourtant, force est de constater que les enjeux sous-tendus par cette pratique dépassent la visée strictement communicationnelle sus-décrite. En quoi le recours massif au jeu de mots s'avère-t-il être une stratégie efficace de communication publicitaire? L'examen d'exemples permettra de mettre en lumière la façon dont le discours publicitaire s'affranchit de certaines contraintes linguistiques en jouant avec les mots sur différents plans de la langue : phonologique, lexical, sémantique ; ces aspects transgressifs illustrant le caractère dynamique inhérent à toute langue. Puis sera envisagée la dynamique animant la construction du sens par le récepteur qui se trouve impliqué et engagé dans ce processus à divers degrés : de sa simple connivence à sa participation active, voire physique dans certains cas, en passant par l'appel à sa culture partagée en vue du décodage de l'intertextualité ou de l'implicite suscités par le jeu de mots. Ce dernier peut d'ailleurs quitter son statut fréquent d'hapax à condition d'être réinvesti dans l'usage. Cela suppose de la part des locuteurs une réappropriation de l'unité lexicale en question pouvant, dans certains cas, conduire au défigement. Ainsi, nous nous interrogerons sur la dimension sociolinguistique qui permet d'envisager le discours publicitaire comme reflet de l'usage linguistique mais aussi comme instigateur de nouvelles tendances linguistiques.

Mots clés : création lexicale, compétence linguistico-ludique, interaction locuteur-récepteur, medium publicitaire, néologisme, production-réception du jeu de mots, transgression, usages du français 


\section{Introduction}

On ne compte plus les Manu Ciao ou autres jeux de mots autour du verbe $\mathrm{Val}(\mathrm{l})$ ser émaillant depuis fin janvier 2017 différents titres de presse ou de billets sur les réseaux sociaux ${ }^{1}$ pour évoquer la défaite du Premier Ministre français Manuel Valls aux élections primaires, preuve s'il en est de la propension de l'actualité à générer ces ludiques trouvailles. D’ailleurs, la diversité des communications lors du colloque La dynamique du jeu de mots - perspectives interdisciplinaires permet de prendre la mesure de cette pratique linguistique qui semble occuper une place de choix dans les différents médias (presse, littérature, réseaux sociaux, publicité). De notre point de vue, nous nous attacherons surtout à examiner le jeu de mots dans un corpus de publicités pour tenter de voir en quoi le recours massif à cette pratique s'avère une stratégie de communication publicitaire efficace. Il s'agira notamment de considérer les trois dynamiques indissociables qu'il génère :

1) au niveau linguistique :

1a) en termes de production, le jeu de mots peut parfois être appréhendé comme une transgression du fonctionnement régulier de la langue ;

1b) en termes de réception, le jeu de mots n'est saisissable par l'interlocuteur qu'à la condition que ce dernier s'engage dans une dynamique d'interprétation ;

2) au niveau sociolinguistique, puisque le jeu de mots est tantôt une création ponctuelle relevant de l'individu, tantôt un objet réinvesti au niveau collectif dans l'usage - voire la langue - et en est le reflet ou l'instigateur de nouvelles tendances.

Mais peut-être nous faut-il, avant d'aller plus loin, expliciter notre titre :

(1) Allumeeez le fun

Nous avons retenu cet exemple en particulier car il nous semblait synthétiser le mieux cette triple dynamique. Il s'agit d'une campagne publicitaire pour l'iPod touch (2012), baladeur numérique d'Apple. D'un point de vue strictement informatif, le message linguistique ne veut pas dire grand-chose au sens où il n'apporte pas de précision particulière sur le produit. L'intérêt est donc ailleurs. D'un point de vue linguistique (et normatif), ce message ne correspond pas à une utili-

1 «Renvoyé Vallser » (Mediapart, 29 janvier 2017) ; « Manu, ciao » (Huffington Post, 29 janvier 2017). 
sation standard de la langue française: la contrainte sémantique n'est pas respectée ; on ne voit pas d'emblée ce qu'allumer le fun pourrait signifier, d'où le fait que cela interpelle le récepteur (fonction phatique). En revanche, un interlocuteur français pourrait trouver cela drôle (ou à tout le moins amusant) car il devrait normalement saisir le clin d'œil de l'annonceur en repérant le jeu phonique (proximité entre /f $\wedge \mathrm{n} /$ et $/ \mathrm{f} ø /$ ) qui lui permet de reconstituer le titre de Johnny Hallyday " Allumez le feu » pour peu qu'il possède cette référence considérée comme faisant partie du patrimoine culturel commun. On voit donc comment le jeu de mots témoigne ici d'une dynamique linguistique (transgression du code), d'une dynamique interactionnelle (connivence entre émetteur et récepteur via l'intertextualité) et, dans une moindre mesure peut-être, d'une dynamique sociolinguistique (réappropriation de l'anglicisme fun, de plus en plus courant en français).

\section{Cadrage théorique et remarques méthodologiques}

À l'instar de Jacqueline Henry pour qui «il ressort de l'analyse des études existantes sur les jeux de mots qu'il est très difficile d'établir une classification définitive des jeux de mots » (Henry 2003 : 30), il serait illusoire de vouloir donner ici une définition absolue du jeu de mots et d'en dresser une typologie exhaustive tant les caractéristiques qui lui sont associées sont polymorphes. L'absence de définition consensuelle autour de ce phénomène n'empêche pas de s'accorder sur sa densité sémantique et Pierre Guiraud formule une première distinction qui permet d'en apprécier les fondements :

[...] le mot d'esprit a pour objet de provoquer le rire, mais joue sur les idées et non sur les mots ; les divertissements verbaux jouent sur les mots - ou plus exactement avec les mots mais n'ont pas pour objet premier de provoquer le rire. C'est donc ce double caractère - une manipulation des mots et qui déclenche le rire - qui constitue l'essence du jeu de mots. (Guiraud 1976 : 104)

Comme le dira plus tard Tzvetan Todorov, « le jeu des mots s'oppose à l'utilisation des mots telle qu'elle est pratiquée dans toutes les circonstances de la vie quotidienne », avant d'ajouter qu' « il voisine avec l'anormal : c'est la folie des mots » (Todorov 1978 : 294), propos auquel nous ne pouvons que souscrire. Ce critère de distorsion par rapport au fonctionnement régulier du système linguistique nous paraît être le premier élément définissant le jeu de mots, même si des 
sous-catégorisations s’avèrent nécessaires. Nous retiendrons simplement ici que les différents plans de la langue peuvent être « affectés » par le jeu de mots. Par ailleurs, au-delà d'une simple transgression volontaire de normes (López Díaz 1996 : 65), Marie-B. Vittoz Canuto (1983 : 29) évoque le caractère dynamique du jeu de mots en tant que lieu de tension entre l'émetteur (qui dispose d'un espace créatif) et le récepteur (appelé à réduire l'anomalie). Elle est en cela rejointe par Jacqueline Henry qui l'envisage comme « un énoncé contenant un ou plusieurs éléments dont la plurivocité a été intentionnellement exploitée par son émetteur » (Henry 2003 : 25). La dimension intentionnelle du jeu de mots nous semble être le deuxième critère à retenir car elle lui donne sa dynamique, en définissant un cadre énonciatif et pragmatique propice à sa production. Dirk Delabastita ne dit pas autre chose puisque, pour lui, le jeu de mots regroupe :

[...] the various discursive phenomena in which certain features inherent in the structure of the language(s) used are mobilised to produce a communicatively significant, (near-) simultaneous confrontation of at least two linguistic units with more or less dissimilar meanings and more or less similar forms. (Delabastita 2011 : 140)

[les différents phénomènes discursifs où sont mobilisées certaines caractéristiques structurales propres à la langue/ aux langues utilisée(s) afin de produire une confrontation (quasi) simultanée et significative sur le plan de la communication entre au moins deux unités linguistiques ayant des significations plus ou moins dissemblables et des formes plus ou moins ressemblantes ; notre traduction]

Ces deux critères définitoires du jeu de mots nous semblent d'autant plus valables s'agissant du domaine publicitaire puisque « [l]e contexte communicationnel extrêmement concurrentiel, caractérisé par un bruit de fond publicitaire, fait $\mathrm{du}$ discours publicitaire un lieu propice aux audaces formelles et aux provocations sémantiques » (Lugrin 2006 : 29). Selon ce même auteur, la propriété innovante de ces transgressions linguistiques doit sans aucun doute se doubler d'un caractère amusant (ou au moins divertissant) puisque « [1]'humour semble être l'un des moteurs les plus efficaces de l'argumentation publicitaire » (Lugrin 2006 : 426) et, partant, quasiment une contrainte de ce type de discours. Les discours publicitaires étant construits par les annonceurs selon cette attente, la question est de savoir si le récepteur saura appréhender et apprécier cette portée ludique.

À ce stade, et sur un plan méthodologique, il convient de préciser que notre corpus n'est pas le fruit d'un recueil systématique de publicités. Il a été constitué pendant plusieurs mois au gré des trouvailles linguistiques. Le colloque et cet article n'ont été qu'un prétexte pour commencer l'étude de quelques-unes de ces 200 occurrences. La totalité des exemples analysés ici sont tirés de publicités contemporaines françaises à vocation commerciale dont l'objet est de vendre des 
produits ou services du quotidien dans des domaines aussi variés que l'alimentaire, la culture, l'hygiène, les transports. Une seule occurrence fait exception à cela : l'exemple (22) où la publicité est alors utilisée à des fins idéologiques afin de faire adhérer à une idée. De plus, tous les exemples mentionnés sont des affiches. Il s'agit d'un support présentant au moins deux spécificités :

- Il permet de combiner des images et de l'écrit, constituants faisant l'objet de disciplines distinctes pour l'analyse. Même si la nôtre portera principalement sur l'aspect linguistique, la réalité plurisémiologique du discours publicitaire est un de ses points forts qui ne saurait évidemment être négligé, ce que nous nous efforcerons de faire dans certains cas où l'appréciation du jeu de mots n'est possible qu'en lien avec le visuel auquel il est associé.

- Il est destiné à être placardé dans les lieux publics. En termes pragmatiques, cela revient à dire que l'intention de son auteur est de donner à voir un produit, une marque, afin d'attirer le regard du plus grand nombre et de déclencher l'achat.

Élément incontournable de communication visuelle, l'affiche s'intègre ainsi au mobilier urbain et investit l'espace public de façon de plus en plus massive, quitte à parfois parasiter sa réception tant les destinataires se trouvent aujourd'hui submergés de messages publicitaires, sous quelle que forme que ce soit (Speck et Elliott 1997). L'objectif est donc de se démarquer par une plus grande dissimulation de la portée commerciale du message au profit d'une plus grande créativité (Rotfeld 2006). Dans ce contexte, la langue (en général) tient un rôle-clé dans ce type de discours persuasif en ce qu'elle véhicule des connotations affectives et émotionnelles qui dépassent le strict aspect communicatif (Holmqvist 2011). On pourra alors s'interroger sur le gain que le jeu de mots (en particulier) permet de retirer dans cette recherche d'efficacité du message publicitaire, notre hypothèse étant que ce dernier constitue une figure linguistique ludique au service d'une stratégie de connivence, le plus souvent humoristique, dont l'intérêt est de situer le récepteur au croisement d'une triple dynamique.

\section{Dynamique linguistique : production du jeu de mots et transgression}

$\mathrm{Au}$ plan phonique, de nombreux jeux de mots se fondent sur une approximation phonétique. Cette exploitation paronymique est la stratégie mise en place par Oasis pour différentes campagnes de promotion de ses boissons fruitées. 
(2) Pour la beauté du zeste (2013)

(3) Ramon Tafraise/Mangue Debol (2014)

L'exemple (2) est le résultat d'une substitution du phonème /3/ à l'initiale par /z/, articulatoirement proche. Cette permutation phonique sur l'axe paradigmatique entraîne aussi une permutation sémantique sur le même axe qui vient renforcer l'effet ludique de ce jeu sur les sonorités puisqu'on obtient alors une paronymie entre geste (allusion ici au geste sportif, qui soutient le visuel de l'affiche où une orange est en train de faire du ski nautique) et zeste (qui s'applique à l'orange). Le même procédé est appliqué au nom des différents personnages de cette campagne. En (3), les permutations respectives du / $/$ / par / / / et du /k/ par /g/ (par voisement pour cette dernière), correspondent dans le même temps à des détournements d'expressions : ramène ta fraise et manque de bol. Le jeu de mots dépasse ici le jeu sur le nom commun pour donner lieu à des pseudo-noms propres.

$\mathrm{Au}$ plan morphologique, la création lexicale ne suit pas systématiquement les procédés classiques de la dérivation et de la composition. Un cas particulier de composition est fréquemment exploité dans le domaine publicitaire: le mot-valise.

(4) complètement rénanové (2012)

(5) REVOLVOLUTION (2010)

(6) L'interro du matin/Bonne à interner/L'international à la loupe/À vous d'intervenir/ L'intermède classique/ Sans interdits (2014)

L'iPod nano a recours à cette formation dans l'exemple (4) où le nom du produit nano se retrouve enchâssé dans l'argument de vente du produit : rénové. Il s'agit en effet d'une version améliorée d'un modèle antérieur. Il en va de même pour le (5) qui est aussi un mot-valise construit par enchâssement. Le nom de la marque (Volvo) est complètement intégré à la caractéristique mise en avant (voiture révolutionnaire). ${ }^{2}$ Parfois même, comme avec la publicité pour France Inter en (6), le nom de la marque et son logotype sont intégrés aux expressions utilisées

2 Il est à souligner que l'iconicisation joue ici son rôle d'aide à la perception de cet enchâssement puisque la couleur utilisée pour les caractères de Volvo est différente des autres. Encore une fois, il ne faut pas oublier que ces messages linguistiques sont supportés par un visuel que nous n'avons pas reproduit ici pour des raisons de droits d'auteur mais qui sont indéniablement à prendre en compte lors d'un examen sémiologique complet. 
pour décliner les programmes de la station. ${ }^{3}$ Jean-Michel Adam et Marc Bonhomme parlent alors d'iconicisation du verbal lorsque les « constituants linguistiques de l'annonce subissent l'influence du domaine iconique. Le langage devient partiellement image. [...] Axée sur le nom, la marque fait partie, comme on l'a vu, du domaine linguistique. Or, les graphèmes qui la composent s'enrichissent souvent secondairement de traits appartenant à la sphère de l'image (iconicisation de la marque) »(Adam et Bonhomme 2012 : 93).

Au plan sémantique, le ressort massivement exploité par le discours publicitaire est le jeu sur la polysémie lexicale et/ou sur l'homonymie syntaxique du français.

(7) Nous aimons choisir avec qui on s'envoie en l'air (2012)

(8) Levez-vous de bonheur (2009)

Dans ces exemples, « il y a à la fois maintien et cassure de la lexie puisqu'à côté d'une valeur figurée admise par le contexte se superpose une valeur propre inhabituelle » (Vittoz Canuto 1983 : 82). L'auteur distingue deux mécanismes du jeu de mots polysémique : «[1] le blocage du sens figuré lexicalisé à la faveur du sens propre, pris au pied de la lettre ; [2] le maintien du sens figuré lexicalisé et la réactivation du sens propre en même temps » (Vittoz Canuto 1983 : 82). Dans l'exemple (7) du comparateur de vols Liligo, ${ }^{4}$ le jeu de mots repose sur la lexie s'envoyer en l'air qui, prise littéralement, signifie aller dans les airs (ce qui semblerait logique pour un annonceur de ce type) mais qui au figuré, et dans un registre très familier, signifie avoir une relation sexuelle. L'aspect ludique ne repose pas tant sur cette ambiguïté linguistique que sur le fait que ce double-sens soit également renforcé par la double-lecture possible du visuel. En effet, le couple censé prononcer cette phrase a l'air passablement " coincé », ce qui déclenche tout d'abord une lecture au sens figuré (décalage qui va susciter l'amusement) mais l'annonceur, représenté sur l'affiche par le pictogramme de l'avion qui décolle, appelle, lui, une lecture au sens propre (ce qui doit a priori faciliter l'élucidation). L'exemple (8) de la marque de jus d'orange Tropicana propose quant à lui une homonymie syntaxique. C'est l'ambiguïté du code oral qui génère

3 Le lecteur pourra se reporter au site suivant pour avoir accès au visuel de cette campagne d'affichage: http://www.radiofrance.fr/l-entreprise/radios-du-groupe/france-inter (dernière consultation le 05/01/2018).

4 Le lecteur pourra se reporter au site suivant pour avoir accès au visuel de cette campagne d'affichage : http://www.creads.fr/blog/tendance-design-graphique/liligo-senvoie-en-lairavec-sa-derniere-campagne-publicitaire (dernière consultation le 05/01/2018). 
ici une perturbation, un brouillage, dans le découpage de l'énoncé, pouvant aboutir tantôt à de bonheur tantôt à de bonne heure. La réalisation phonétique de ces deux segments est absolument identique. L'équivoque n'apparaît qu'au moment de la transcription graphique, laquelle permet l'élucidation sémantique. Ce procédé est extrêmement fréquent dans des langues comme le français où « le phénomène de liaison [...] par lequel les mots s'enchaînent, s'emboîtent, tout en se modifiant légèrement, facilite l'apparition d'ambiguïtés générées par l'effacement de la frontière composant un syntagme » (Vittoz Canuto 1983 : 34).

Ces divers exemples révèlent donc bien le caractère dynamique de la langue qui, loin d'être aussi figée que d'aucuns le pensent, ${ }^{5}$ est tout à fait malléable, entre autres par les annonceurs, qui exploitent les moindres potentialités de son système, au risque parfois de s'éloigner du fonctionnement régulier et de créer certaines anomalies. De toute évidence, ce sont bien ces dernières qui fonctionnent comme autant de signaux pour le récepteur. Une telle dimension phatique est l'un des principes de base de la publicité : avant de vendre un produit ou de faire adhérer à une campagne, encore faut-il attirer l'attention de l'acheteur potentiel.

\section{Dynamique sémantique : réception du jeu de mots et construction du sens}

Une fois l'attention attirée par la détection d'une anomalie, le mécanisme à l'autre extrémité de la chaîne de communication consiste, d'après nous, en la réduction de la tension créée par cet écart. Cela suppose bien évidemment une participation plus ou moins active du récepteur qui se trouve alors impliqué et engagé dans ce processus à des degrés divers.

En matière de culture partagée d'abord, afin que l'effet humoristique escompté se réalise, le jeu de mots se doit d'être facilement repérable, puis interprétable par l'interlocuteur ou que ce dernier soit capable de lever lui-même les éventuelles ambiguïtés ainsi générées. ${ }^{6}$ L'humour étant un levier d'adhésion,

5 Les partisans d'une conception passéiste de la langue, qu'ils soient «simples " locuteurs, académiciens ou grammairiens, fondent leur position sur la défense d'une belle langue, indépendamment de toute approche scientifique des questions linguistiques dont ils refusent par ailleurs de reconnaître la légitimité (Dubois 2014).

6 Nous entendons ici le terme d'ambiguïté au sens que lui donnent les linguistes de « résidu irréductible, qui appelle plusieurs représentations métadiscursives disjointes [...] on sait l'usage abondant que la publicité fait de ce type de ressource » (Fuchs 2009 : 7-10). 
un message drôle crée normalement une émotion chez le consommateur : sinon le rire dans le cas de l'affiche, au moins le sourire, témoin de l'instauration d'une connivence. Dans le cas «idéal » où les connaissances encyclopédiques, culturelles et / ou sociales du récepteur correspondent en tous points à celles de l'émetteur, ${ }^{7}$ il n'y a pour ainsi dire aucun problème de réception, à tel point que l'énonciateur peut même aller jusqu'à une fragmentation partielle ou totale du message :

(9) Have a br/have a (2014)

(10) ingle ells, ingle ells (années 70)

Ici, le récepteur "averti » est tout à fait capable de reconstituer le nom de la marque J\&B en (10) ainsi que la moitié manquante du slogan de KitKat en (9) : Have a break, have a KitKat. Le jeu de mots ne fonctionne qu'à la condition qu'il $\mathrm{y}$ ait cette interaction/dynamique entre les différents acteurs de la communication. Il faut en effet que le récepteur soit familier avec le slogan de la marque KitKat dans l'exemple (9), et qu'il ait repéré qu'il s'agissait des paroles de la chanson Jingle Bells dans l'exemple (10). Dans le premier cas, sa connaissance encyclopédique est sollicitée par l'aspect graphique (on retrouve les couleurs caractéristiques de la marque, ainsi que la troncation de l'affiche qui suggère que le colleur d'affiche a lui-même pris une pause pour savourer son KitKat). Dans le second, sa connaissance encyclopédique lui permet de déduire le nom du produit. C'est parce qu'il repère l'absence répétée des lettres J et B qu'il arrive à reconstituer le nom du produit. En ce sens, «la publicité contribue largement à convoquer le lecteur, en lieu et place du consommateur, comme partenaire dans cette aire de jeu. L'argumentation publicitaire opère dès lors plutôt sur le registre de la séduction, de la surprise, du divertissement et de la complicité que sur celui de la persuasion » (Lugrin 2006 : 17). Précisément, une telle complicité entre partenaires de la communication publicitaire dépendra fortement de prérequis : 1) des savoirs que l'émetteur prête à son interlocuteur et qu'il suppose partagés et 2) des connaissances encyclopédiques et acquis socio-culturels effectifs du récepteur et de la capacité de ce dernier à « filtrer et sélectionner ceux qui conviennent à la situation » (Lugrin 2006 : 79), ce qui conduit à penser que «la culture fonctionne bien comme un facteur de connivence [et que le slogan] opère une jonction entre son auteur (supposé) et ses lecteurs ciblés (virtuels) qui sont censés partager les mêmes goûts, les mêmes connaissances, la même culture, les

7 On sait, depuis Galisson (1988), que cela reste un idéal et que les différents niveaux de connaissance sont propres à chaque individu. 
mêmes représentations » (Sautot 2000 : 30). Beaucoup de paramètres susceptibles d'avoir un impact interviennent donc sur la dynamique production-réception du jeu de mots et sa réussite (ou son échec). ${ }^{8}$ En effet, en publicité, l'une des formes les plus répandues d'intertextualité est le recours à l'allusion. ${ }^{9}$ La composante explicite du message se double alors d'une dimension implicite (partie connotative) qu'il convient de saisir pour pleinement apprécier le jeu de mots, d'autant plus que le « jeu de mot in absentia [se fait] sur l'axe paradigmatique et [qu'il] fonctionne alors sur l'évocation d'une connotation le plus souvent remaniée par le concepteur » (Vittoz Canuto 1983 : 101). Quelques exemples illustrent ce phénomène :

(11) La pelle du 18 juin (2013)

(12) Sous les pépins la plage (2013)

(13) On va fluncher! (années 90)

(14) Scallaboosh, Scallaboosh, will you do the banned tango (2008)

(15) Avec l'application Transilien, un homme azerty en vaut deux (2011)

(16) La France, à ce prix-là je la quitte ! (2008)

On voit que l'intertextualité revêt plusieurs formes. Elle peut renvoyer à :

- des épisodes historiques : en (11), Jardiland, par le biais de l'homonymie syntaxique décrite plus haut, fait clairement référence (soutenue en cela par l'iconographie de l'affiche) à ce célèbre épisode où le général de Gaulle appelle (l'appel vs. la pelle) les Résistants français à continuer la lutte contre les Nazis. Or, l'objet de la publicité est, beaucoup plus prosaïquement, de faire venir le client dans les jardineries de l'enseigne afin de l'équiper, à l'arrivée des beaux jours, en accessoires de jardinage... Idem pour l'exemple (12) de la marque de jus de fruits Oasis qui fait référence à un slogan solidement ancré dans l'inconscient culturel français : Sous les pavés la plage est en effet

8 Si tant est que l'on puisse effectivement et objectivement «mesurer » l'un ou l'autre. Au sujet de l'évaluation du jeu de mots, le lecteur se reportera à la contribution de Catherine KerbratOrecchioni (ce volume).

9 D’après la distinction établie par G. Genette entre les différentes formes d'intertextualité que sont la citation, le plagiat, la référence et l'allusion, cette dernière «[faisant] référence de manière plus ou moins lâche à un texte ou un ensemble de textes antérieurs sans en expliciter la source. Relevant d'une certaine subjectivité, elle peut ne pas être perçue, ou l'être là où elle ne se trouve pas » (Lugrin $2006: 205)$. 
l'un des slogans qui symbolise les aspirations de liberté prônées par les manifestants en mai 68 ;

- des œuvres antérieures issues de domaines divers : littérature, musique, chanson, cinéma... À propos de la chanson, on peut penser à la chaîne de restauration à volonté Flunch qui a détourné une chanson populaire de Gilbert Montagné (On va s'aimer) pour faire passer son slogan (13), produisant au passage un néologisme morphologique. ${ }^{10}$ Nous évoquions plus haut le recours à la séduction comme stratégie publicitaire. Avec l'exemple du Hard Rock Café cherchant à vanter ses soirées karaoké (14), la connivence entre annonceur et récepteur est poussée à l'extrême puisque le publicitaire, par un jeu de polyphonie, ${ }^{11}$ se met à la place de son client qui « fait du yaourt », de l'à-peu-près lorsqu'il essaye de chanter une chanson dont il ne connaît pas ou pas bien les paroles. Tout le monde peut ainsi s'identifier à ce récepteur. La séduction s'opère également sur la capacité à reconnaître in fine le titre de la chanson en question : Bohemian Rhapsody de Queen. En y parvenant, la compétence linguistico-ludique du locuteur-récepteur s'en trouve flattée. On voit alors très bien comment le plaisir causé par le jeu est notoire et s'inscrit dans une double perspective. En effet, annonceur et récepteur en jouissent tous deux : le premier en se satisfaisant de son clin d'œil qui atteste de son habileté à déconstruire la langue afin qu'elle soit mieux reconstruite par le second, qui éprouve, ce faisant, une certaine satisfaction intellectuelle. ${ }^{12}$ Ce plaisir partagé aussi bien par l'un que par l'autre témoigne donc d'une dynamique incessante conduisant Camille Vorger (2011) à définir le pacte colludique, notion qui consiste en la recherche d'une certaine complicité entre l'auteur du jeu de mots et son public ;

- des proverbes ou expressions figées, comme dans l'occurrence (15) où le jeu consiste en la substitution de l'adjectif averti (du proverbe initial) par le nom du clavier Azerty (la base de l'utilisation d'une telle application étant le clavier de smartphone ou d'ordinateur qui permet précisément d'être averti). Il serait facile de multiplier les exemples de ce genre d'allusions tant la proximité formelle entre proverbes et slogans favorise leur rapprochement : «[ils] connaissent une fraternité formelle indéniable, qui facilite et encou-

10 Procédé de dérivation fréquent en français qui permet de passer d'un nom de marque souvent emprunté à l'anglais - à un verbe, comme pour googler, liker, scotcher, stabiloter, karchériser, etc.

$11 \mathrm{Au}$ sens où Ducrot l'entend, c'est-à-dire « [lorsqu'] on peut distinguer dans une énonciation deux types de personnages, les énonciateurs et les locuteurs » (Ducrot 1984 : 193).

12 N'omettons pas, cependant, que le jeu de mots est utilisé en publicité à des fins de vente, persuasion et adhésion avant tout (rarement en tant que tel, dans sa seule dimension ludique). 
rage leur mise en relation » (Lugrin 2006 : 228). L'exemple du défigement est à ce propos éclairant : lorsqu'une lexie a été tellement entendue et réutilisée par les locuteurs, elle s'ancre telle quelle dans la mémoire collective et se prête alors d'autant plus facilement à un détournement, un défigement. « La publicité exploite [d'ailleurs] 'à chaud', suivant le procédé de la parodierebond, la résonance médiatique extrême qu'ont eue certains slogans lors d'élections présidentielles » (Adam et Bonhomme 2012 : 202). C'est le cas en (16) avec le slogan d'accroche de la compagnie low-cost Transavia, où l'allusion à la phrase très controversée de Nicolas Sarkozy à propos de l'immigration choisie lorsqu'il était candidat à la présidentielle en 2007, La France tu l'aimes ou tu la quittes, est plus qu'évidente. ${ }^{13}$ On rejoint alors Charlotte Schapira qui observe à propos de la déproverbialisation des proverbes que, contre toute attente, «[...] le détournement consolide le proverbe détourné, dans l'usage, autant que l'emploi de la forme standard» (Schapira 2000 : 95). Le discours publicitaire peut, dans ce cas, être envisagé aussi comme un véritable « relais culturel » (Lugrin $2006: 37$ ).

On voit donc que «[ces jeux de mots] basés sur des connotations allusives fonctionnent différemment : non plus [tant] sur le langage lui-même mais sur le rappel à un savoir antécédent présent dans la mémoire des locuteurs récepteurs. [...] L'allusion joue sur l'inconscient beaucoup mieux que n'importe quel autre type de jeux de mots en détournant la vigilance de l'esprit critique » (Vittoz Canuto 1983 : 114-115). Néanmoins, la vigilance a beau être détournée, il n’en reste pas moins que le locuteur-récepteur est sollicité plus ou moins directement dans la construction du sens. Cela apparaît de manière tout à fait flagrante dans une publicité de la Française des Jeux où le visuel témoigne clairement de la double possibilité sur l'axe des choix (paradigmatique) qui s'offre au récepteur et qui lui donne par la même occasion une double-lecture sur l'axe horizontal (syntagmatique). ${ }^{14}$ À lui évidemment de «choisir », de décider de telle ou telle interprétation : dans un cas, il gagne gros, dans l'autre il ne gagne rien. Mais les deux options restent envisageables linguistiquement et sémantiquement parlant

13 Même si ce ne sont pas les propos exacts qu'il a tenus : "S'il y en a que ça gêne d'être en France, je le dis avec le sourire mais avec fermeté, qu'ils ne se gênent pas pour quitter un pays qu'ils n'aiment pas » (Soir 3 du 23 avril 2006, INA). Le raccourci qui s'était ensuivi avait d'ailleurs fait l'objet d'une vive polémique.

14 Les visuels en question jouent sur les mots en présentant ces derniers deux à deux sur le rouleau d'une machine à sous (où s'affichent habituellement les symboles du jackpot). La suggestion est qu'un gain au loto permet ainsi de passer de «cassoulet » à « cabriolet »; de « coquillettes » à « coquillages »; de « travail » à « transat ». 
(c'est le principe du jeu de hasard que symbolise la Française des Jeux). C'est au locuteur de construire le sens.

Enfin, l'implication du locuteur-récepteur dans le décodage de l'information est maximale lorsque celui-ci est censé être physiquement présent pour avoir accès au dénouement - ludique - d'une campagne. Ainsi, en 2010, une campagne d'affichage a été lancée sur le principe d'un teasing en 3 temps. D'abord, des affiches montraient des visages d'hommes et de femmes ravis et épanouis surplombant une accroche allusive :

(17) Aujourd'hui je l'ai fait (2010)

Fait quoi ? se demande aussitôt le récepteur qui, en associant le visuel et le linguistique, aura tôt fait de voir dans ce message une connotation sexuelle. Il n'en faut pas plus pour piquer sa curiosité et le rendre alors beaucoup plus attentif à la phase de teasing suivante. Quelques jours plus tard, une nouvelle étape du teasing a mis en avant des variantes du message initial, les affiches reposant, elles, toujours sur le même visuel :

(18) Aujourd'hui je l'ai fait et j'y pense encore/ et c'était très fort/avec un très bon ami/pour de l'argent (2010)

La première intuition du récepteur se voit - selon la variante envisagée - renforcée ou invalidée. Arrive alors la troisième vague d'affiches qui permet de découvrir ce que ces personnes ont bien pu faire de sensationnel pour les mettre dans des états pareils. Elles ont " seulement » ouvert un compte en ligne à la banque ING Direct... Ici, le jeu ne provient assurément pas des mots eux-mêmes mais précisément de leur absence, du non-dit. Un jeu de non-mots, en somme. Tout repose sur la suggestion, l'allusion. L'engagement du récepteur est alors fondamental ici, et même physique puisque s'il veut pouvoir construire le sens et saisir la portée comique (ou ne serait-ce que le message informatif) de cette publicité, il doit être effectivement présent dans le temps et dans l'espace, ${ }^{15}$ engagement total donc.

15 Si l'on n'est pas physiquement présent à l'une ou l'autre des phases de teasing et si l'on ne cherche pas à passer dans le même lieu à plusieurs jours d'intervalle, il manque une clé de lecture pour accéder à la portée ludique du message. 


\section{Dynamique sociolinguistique : le jeu de mots comme création ponctuelle ou réinvestisse- ment dans l'usage}

Au-delà du cadre strictement linguistico-sémantique qui confère à ces réalisations hors-normes un statut particulier, nous pouvons aussi nous interroger sur leur portée sociolinguistique, au sens premier et très général du terme où la langue est envisagée comme un produit social et n'existe que parce que les locuteurs intériorisent son existence via leurs pratiques (Blanchet et Bulot 2013). Bien qu'étant le plus souvent des créations ponctuelles, ces jeux de mots dans le discours publicitaire fonctionnent à la fois comme des témoins de l'usage en évolution, mais aussi comme des instigateurs de nouvelles tendances linguistiques (Bonhomme 2002).

Dans le premier cas, l'examen d'un certain nombre d'affiches laisse à penser que la publicité tient un rôle de «fixateur » de la dynamique linguistique. Elle photographie pour ainsi dire, à un instant $t$, l'usage dans toutes ses manifestations, même les plus écartées de la norme. ${ }^{16}$ L'exemple suivant de mots non attestés dans la langue, pas plus que dans l'usage, illustre ce phénomène :

(19) attractionisme/sublimitude/ bombassitude/rayonescence/glamourisme/fascinance (2013)

Ces créations morphologiques, obtenues par manipulation des possibles de la langue française, ne constituent pour l'instant que des hapax, au sens où ces innovations - même si leur portée néologisante est à nuancer (Favreau 2015 : 200) - n'ont à notre connaissance pas été reprises (Anokhina 2003) et constituent en cela des créations morphologiques «de circonstance » (Dal et Namer 2018 : 215). C'est incontestablement ce côté « inattendu, paradoxal de l'association des formants des néologismes, d'un point de vue sémantique ou morphologique » (Sablayrolles 2015 : 201) qui leur donne un aspect ludique et permet qu'on les qualifie de jeux de mots. Exception faite de ces innovations très ponctuelles qui font toutefois montre des potentialités infinies de notre système linguistique,

16 C'est une acception interactionnelle de sociolinguistique qui permet ce commentaire. Depuis Gumperz, la langue est envisagée comme diverse et hétérogène, l'usage variant localement et / ou socialement selon les types d'interaction qui revêtent un rôle essentiel dans la construction d'identités sociales multiples imposant d'analyser la langue dans sa dimension sociale pour appréhender l'influence réciproque entre les faits linguistiques et sociaux (Gumperz 1989). 
certaines anomalies morphologiques éphémères "peuvent influencer et même encourager une certaine mode linguistique » (Vittoz Canuto 1983 : 122). Il en va ainsi de la tendance croissante à utiliser le substantif comme un adjectif $:^{17}$

(20) Restez nature (2011)

(21) Tellement plus pep's! (2016)

Dans ces exemples pour le savon Petit Marseillais (20) et pour un centre commercial (21), les substantifs nature et pep's sont employés comme adjectifs.

Si l'on tend un peu l'oreille, on se rend compte que ce procédé de dérivation impropre, qui consiste à employer un terme dans une autre catégorie grammaticale que la sienne, teinte de plus en plus les discours du quotidien. Chez nos étudiants (mais pas uniquement), la montée en puissance depuis quelques années du juste, adjectif utilisé comme adverbe, est à cet égard éloquente. Il n'est pas rare d'observer ce calque syntaxique de l'anglais ${ }^{18}$ où l'adjectif est mis en lieu et place de son équivalent adverbial tout simplement pour souligner l'emploi de l'adjectif attribut du sujet. Une campagne de la Prévention Routière a d'ailleurs surfé sur cette mode et proposé, dans un registre un peu grinçant :

(22) Vous avez juste oublié un clignotant, il est juste un peu mort (2007)

On peut ici percevoir une nuance entre les deux emplois de cet adverbe. Dans le premier segment, juste peut être aisément remplacé par seulement, cette valeur restrictive découlant directement du sens originel de juste (= avec exactitude). Cet emploi est alors justifié. Dans le second segment, juste peut être remplacé par tout simplement, octroyant à cet emploi une valeur adverbiale euphémisante, renforcée d'ailleurs par le "un peu» qui le suit. De cette accumulation de procédés provient le décalage ludique puisque « mort » fait partie de cette catégorie de verbes perfectifs qui font qu'on ne peut pas être « un peu » mort. ${ }^{19}$ Cette co-occurrence insolite de deux emplois (l'un ancien, l'autre plus récent) d'un terme au sein d'un même slogan illustre la façon dont le discours publicitaire se fait alors miroir de nouveaux usages, ce qui ne peut contribuer, nous semble-t-il, qu'à dynamiser davantage ces modes linguistiques avant de les installer, peut-

17 Cela est probablement à mettre sur le compte du phénomène de contact des langues, notamment avec l'anglais (Paillard 2000).

18 He's just amazing.

19 Cela fait référence, en termes de logique aristotélicienne, au tertium non datur, où une proposition $p$ et sa négation non- $p$ ne peuvent être vraies en même temps, ce qui est évidemment le cas pour mort / vivant ; il n'existe pas de troisième solution. 
être, dans la langue, au risque qu'elles «ne so[ie]nt plus perçues comme plaisantes, drôles, créatives, etc. » (Winter-Froemel 2016 : 263) en devenant usuelles. Récemment, l'enseigne de produits culturels Fnac a, sur ses affiches, prêté les mots suivants à diverses personnalités du monde de la culture :

(23) J'ai fait la Fnac (2015)

Cet emploi transitif de faire fait ici écho à faire une grande école (= aller dans une grande école). On fait donc la Fnac comme on ferait l'ENA, les Beaux-Arts, une Fac de Droit. De là à penser qu'on en sort avec les mêmes aptitudes, il n’y a évidemment qu'un pas que les publicitaires attendent de voir franchi par de futurs clients.

Non seulement la publicité est le reflet de pratiques linguistiques plus ou moins innovantes et ludiques, mais elle est parfois également elle-même à l'origine de nouvelles conduites en matière d'usage linguistique.

(24) Avec Carrefour je positive (1988)

(25) Volcanisez-vous (2013)

Il y a 30 ans, l'enseigne Carrefour lançait un nouveau slogan pour ses magasins (24) où le verbe positiver, formé par dérivation de l'adjectif positif, connaît un nouvel emploi syntaxique. Jusqu'alors uniquement connu dans un emploi transitif, ${ }^{20}$ il adopte désormais une construction également intransitive, emploi d'un usage tout à fait courant aujourd'hui, à tel point qu'il a même été fixé par certains dictionnaires de langue. ${ }^{21}$ De même, le procédé de construction à l'origine de cette création singulière s'est lui aussi perpétué et on retrouve ainsi l'apparition d'un verbe inattendu et inédit en (25) pour l'eau minérale d'origine volcanique Volvic, qui a sans doute plus vocation à interpeller qu'à être réinvesti par les locuteurs (tout comme fluncher, cité précédemment). En d'autres termes, il s'agit là de mettre l'accent sur la singularité d'un message pour lui permettre de gagner en visibilité par rapport aux autres dans un « contexte communicationnel extrêmement concurrentiel» (Lugrin 2006 : 29). Enfin, on est à même de constater une tendance qui se fait jour depuis peu : les locuteurs eux-mêmes s'improvisent parfois publicitaires sur les réseaux sociaux. En effet, il existe un jeu qui y fait fureur et qui consiste pour l'individu à générer des jeux de mots à partir des noms de marques ou de produits de son environnement immédiat, à photographier ses

20 Cet emploi est attesté dès 1839 dans le TLFi.

21 Le Larousse donne cet exemple d'emploi intransitif : dans la vie il faut positiver. 
productions, et à les poster sur les réseaux dans l'attente de réactions de ses concitoyens. ${ }^{22}$ Le logo Darty se voit ainsi affublé de deux petits papiers : un devant portant l'inscription feu et l'autre derrière avec la mention fice ; le logo Krys est précédé d'un petit papier où il est écrit Jésus et ainsi de suite. La dimension esthético-ludique - et gratuite - d'une telle dynamique prévaut dans ce cas sur la visée publicitaire (c'est-à-dire phatique et persuasive) qu'ont les annonceurs, même si la diffusion large et étendue de ces créations contribue indéniablement à leur publicité. Cela témoigne aussi de l'appétence certaine qu'ont les locuteurs lambdas pour jouer avec les mots. En devenant eux-mêmes producteurs de jeux de mots publicitaires, ils expriment leur capacité métalinguistique, créative et récréative qui est au moins aussi importante que leur aptitude à se poser en récepteurs des jeux de mots. En somme, la cible devient le publicitaire ; la boucle est donc bouclée ! Marshall McLuhan, pour qui « [l]e message, c'est le médium » (McLuhan [1964] 1968 : 23), ne l'aurait pas dit autrement, évoquant la primauté de la nature même du médium sur le contenu du message à proprement parler.

\section{Conclusion}

$\mathrm{Au}$ terme de cette contribution, il apparaît d'abord que le discours publicitaire semble reposer massivement sur une composante ludique se manifestant sur différents plans (tant visuel que linguistique), si bien que « [1]a publicité n'est plus un dialecte marginal, mais un supra-langage [...] peut-être la langue la plus vivante aujourd'hui... » (Cathelat [1968] 1987 : 238). De plus, le jeu de mots du discours publicitaire génère une dynamique où se croisent et se superposent plusieurs niveaux : linguistique d'abord en ce qu'il exploite les ressources de la langue pour aboutir à des productions, sinon inédites, au moins toujours curieuses et à même de libérer l'expression ; sémantico-pragmatique ensuite dans la mesure où l'interaction et la connivence apparaissent comme essentielles au processus de décodage de l'intertextualité et partant, de l'accès au sens; sociolinguistique enfin puisque le discours publicitaire fait partie intégrante du quotidien des locuteurs et fonctionne à ce titre comme un miroir des usages et changements linguistiques et sociolinguistiques tout en étant parfois aussi luimême source d'innovations, reprises parfois par la communauté de locuteurs. En situant ainsi le récepteur à la confluence de trois dynamiques, il en résulte que ce

22 Le jeu s'appelle «Laissez parler les petits papiers » et on peut trouver une multitude d'exemples sur plusieurs réseaux sociaux via le hashtag \#LPLPP. 
dernier se voit assigner un rôle accru dans l'interaction, l'invitant à s'engager pleinement dans les processus dynamiques de production, d'interprétation et de réinvestissement. La question fondamentale des critères de réussite du jeu de mots reste néanmoins posée. Quand Gilles Lugrin dit que «l'humour semble être l'un des moteurs les plus efficaces de l'argumentation publicitaire » (Lugrin 2006 : 426), on ne peut que partager son point de vue mais quelles données objectives et empiriques permettent d'affirmer cela ? Ce questionnement a fait l'objet, en partie, d'une contribution à ce volume par Catherine Kerbrat-Orecchioni, vers laquelle nous renvoyons volontiers le lecteur pour prolonger sa réflexion.

\section{Références bibliographiques}

Adam, Jean-Michel \& Marc Bonhomme. 2012. L'argumentation publicitaire. Paris : Armand Colin.

Anokhina, Olga. 2003. La contribution des noms abstraits à la formation des néologismes. In Jean-François Sablayrolles (éd.), L'innovation lexicale, 437-455. Paris : Champion.

Blanchet, Philippe \& Thierry Bulot. 2013. Une introduction à la sociolinguistique pour l'étude des dynamiques de la langue française dans le monde. Paris : Éditions des archives contemporaines.

Bonhomme, Marc. 2002. La publicité comme laboratoire du français actuel. L'information grammaticale 94. 33-38.

Cathelat, Bernard. [1968] 1987. Publicité et société. Paris : Payot.

Dal, Georgette \& Fiammetta Namer. 2018. Playful nonce-formations in French : Creativity and Productivity. In Sabine Arndt-Lappe, Angelika Braun, Claudine Moulin \& Esme WinterFroemel (éds.), Expanding the lexicon. Linguistic Innovation, Morphological Productivity, and Ludicity (The Dynamics of Wordplay 5), 203-228. Berlin \& Boston : De Gruyter.

Delabastita, Dirk. 2011. Wholes and holes in the Study of Shakespeare's Wordplay. In Jonathan Culpeper \& Mireille Ravassat (éds.), Stylistics and Shakespeare's Language : Transdiciplinary Approaches, 139-164, London : Continuum.

Dubois, Vincent. 2014. Le rôle des linguistes dans les politiques de la langue française (19601990) : éléments pour une analyse socio-politique. Dossiers d'HEL [Histoire Epistémologie Langage], SHESL, Linguistiques d'intervention. Des usages socio-politiques des savoirs sur le langage et les langues, 6. http://dossierhel.hypotheses.org/. halshs-01115127 (dernière consultation le 05/01/2018).

Ducrot, Oswald. 1984. Le dire et le dit. Paris : Minuit.

Favreau, Hélène. 2015. «On s'keep in Sosh » ou l'exemple du français libéré dans et par la publicité. Voix Plurielles 12(1). 197-206.

https://brock.scholarsportal.info/journals/voixplurielles/article/view/1185/1111 (dernière consultation le 22/02/2017).

Fuchs, Catherine. 2009. L'ambiguïté : du fait de langue aux stratégies interlocutives. Travaux neuchâtelois de linguistique 50. 3-16.

Galisson, Robert. 1988. Cultures et lexicultures. Pour une approche dictionnairique de la culture partagée. Cahiers d'Études Hispaniques Médiévales. 325-341. 
Guiraud, Pierre. 1976. Les jeux de mots. Paris : Presses Universitaires de France.

Gumperz, John. 1989. Engager la conversation. Introduction à la sociolinguistique interactionnelle. Paris : Minuit.

Henry, Jacqueline. 2003. La traduction des jeux de mots. Paris : Presses Sorbonne Nouvelle.

Holmqvist, Jonas. 2011. Consumer language preferences in service encounters : A crosscultural perspective. Managing Service Quality 21.178-191.

INA. 2006. Journal télévisé Soir 3 du 23 avril 2006. https://www.ina.fr/video/3073038001004 (dernière consultation le 05/01/2018).

López Díaz, Montserrat. 1996. Écriture publicitaire : ludisme et infractions. La linguistique 32. 65-79.

Lugrin, Gilles. 2006. Généricité et intertextualité dans le discours publicitaire de presse écrite. Berne : Peter Lang.

McLuhan, Marshall. [1964] 1968. Pour comprendre les médias : les prolongements technologiques de l'homme, traduit de l'anglais par Jean Paré. Paris : Seuil.

Paillard, Michel. 2000. Lexicologie contrastive anglais-français : formation des mots et construction du sens. Gap : Ophrys.

Rotfeld, Herbert Jack. 2006. Understanding advertising clutter and the real solution to declining audience attention to mass media commercial messages. Journal of Consumer Marketing 23(4). 180-181.

Sablayrolles, Jean-François. 2015. Néologismes ludiques : études morphologique et énonciativo-pragmatique. In Esme Winter-Froemel \& Angelika Zirker (éds.), Enjeux du jeu de mots. Perspectives linguistiques et littéraires (The Dynamics of Wordplay 2), 189-213. Berlin \& Boston : De Gruyter.

Sautot, Jean-Pierre. 2000. Lire dans l'espace urbain : les paradoxes des enseignes commerciales. Langage et société. 29-44.

Schapira, Charlotte. 2000. Proverbe, proverbialisation, déproverbialisation. Langages 139. 81-97.

Speck, Paul \& Michael T. Elliott. 1997. The antecedents and consequences of perceived advertising clutter. Journal of Current Issues \& Research in Advertising 19(2). 39-54.

Todorov, Tzvetan. 1978. Les genres du discours. Paris : Seuil.

Vittoz Canuto, Marie-B. 1983. Si vous avez votre jeu de mots à dire. Analyse de jeux de mots dans la presse et dans la publicité. Paris : Nizet.

Vorger, Camille. 2011. Poétique du slam : de la scène à l'école. Néologie, néostyles et créativité lexicale. Thèse sous la direction de Francis Grossmann et Dominique Abry. Université de Grenoble. https://tel.archives-ouvertes.fr/tel-00746972 (dernière consultation le 09/06/2018).

Winter-Froemel, Esme. 2016. Les créations ludiques dans la lexicographie et dans l'interaction locuteur-auditeur : aspects structurels, enjeux sémantiques, évolution diachronique. In Christine Jacquet-Pfau \& Jean-François Sablayrolles (éds.), La fabrique des mots français, 251-267. Limoges : Lambert-Lucas.

\section{Usuels}

Petit Larousse. 2011. Paris : Larousse.

TLFi : Trésor de la Langue Française informatisé. http://www.atilf.fr/tlfi, ATILF - CNRS \& Université de Lorraine. 

Appendice 
